\title{
Wearable bio-sensors: a gigantic leap in health care system
}

\begin{abstract}
This concise communication overviews the development of wearable bio-sensors. It outlines the basics as well as emerging development of wearable bio-sensors. Apart from this, the recent development in this filed comprehensively analyzed along with future recommendations.
\end{abstract}

Volume 6 Issue 4 - 2020

\author{
Biswas R \\ Department of Physics, Tezpur University, India
}

Correspondence: Biswas R, Department of Physics, Tezpur University, Applied Optics and Photonics Lab, Tezpur-784028, Assam, India, Tel +91-037|2-275564, Fax +91-37|2-267-005, Email rajb@tezu.ernet.in

Received: October 30, 2020 | Published: November 17, 2020

\section{Introduction}

Sensors play a very vital role in the wellbeing of human lives. From daily household activities to industrial process, sensors are the part and parcel for continuous and sustainable development. Spanning a wide range of fields such as chemical, physical as well as biophysical; it has become ubiquitous. As for instance, numerous sensors and sensory schemes have been reported for assessing temperature; humidity; pressure; heavy-metal ion sensing as well as in various healthcare systems. ${ }^{1-12}$ When we talk about a sensor; the point-of-care approach is always preferred. Accordingly, the healthcare industry is slowly beaming with innovative concepts. This concise communication outlines the transition phases of sensors used in health care system. Apart from this, it also briefs the recent progress in the area of printable sensors. The transition phases that the sensing schemes are now undergoing is well displayed in Figure 1. As rightly pointed out in Figure 1, earlier sensors used to be bulky as well as very expensive. Hence, they were not portable as well as sensitivity also remained very low. With growing demand in healthcare system, there had been some radical changes such as portability as well as superior detection ability. However, they have not been adequate to mollify the growing health industry. Of late; miniaturization with minimal accessories has become the need of the hour. Slowly, rigid as well as voluminous sensory schemes are becoming obsolete nowadays as everyone is more accustomed to hassle free and comfy wears. Taking cue from this, manufactures have now resorted to making wearable devices such as smart watches, fit-bit etc.

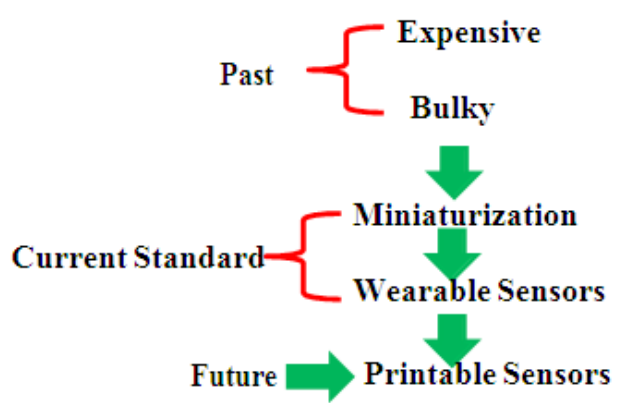

Figure I Transitions phases of sensors in healthcare.

which not only appraises the users about time but also let them know about their health conditions. In course of time, this field of wearable electronics has been booming with newer progress. With the advent of sophisticated cutting edge technology, experts are now gearing up to revolutionize the wearable industry pertaining to healthcare systems. As for instance, we can talk of dermal tattoos functioning as bio sensor for various health ailments. Researchers from Imperial College of London had been instrumental in designing colorimetric biosensors for metabolite detection measuring a wide range of variables such as $\mathrm{pH}$, glucose as well as albumin concentration with further scope of being utilized in diabetes, liver failure etc. ${ }^{13}$ In another recent development, Yetisen et $\mathrm{al}^{14}$ devised fluorescent dermal tattoo biosensors for electrolyte analysis. Aided by smart phone algorithms, this sensory scheme has the potential of continual assessment of hydration status in point-of-care settings. ${ }^{14}$ Simultaneously, there is another emerging field in the area of printable electronics. With the aid of state-of-art 3D printer, it has been possible to print sensors directly on surfaces. Now, researchers are printing sensors directly on human skin without the use of heat. As we know that even small components in a circuit has to be soldered into its respective places. This process of soldering also leads to generation of heat. Hence, printing directly or pasting a whole circuit requires special treatment. Very recently, researchers developed a simple fabrication technique. ${ }^{15}$ Researchers previously developed flexible printed circuit boards for use in wearable sensors; but, printing directly on skin has been an obstacle. The sintering process requires temperatures of about $300^{\circ} \mathrm{C}$ to bond the sensors silver nanoparticles together. However, such high temperature is beyond tolerance for human skin. In order to sort out this, researchers devised a scheme where they incorporated another sintering aid layer. The aim was to reduce the temperature during the overall sintering process. With the addition of nanoparticle to the mix, the silver particles start to sinter at a reduced temperature which is $100^{\circ} \mathrm{C}$. This process facilitates in printing sensors in cloths as well as papers. However, this was not enough. It is imperative to further narrow down the temperature so that it could be executed at room temperature. Accordingly, researchers changed the formula of the aid layer so that it helps the sintering achievable at room temperature. The newly modified layer comprises of polyvinyl alcohol paste. This produces immediate beneficial effects. First, it minimizes the roughness of the printing surface. Apart from that, an ultra-thin layer with metallic patterns can be amenable giving flexibility as well as fold ability. Above all, the electrochemical capabilities of the ultra-thin layer remain intact. The sensors are capable of recording temperature with high precision. It also aids in sensing humidity, oxygen levels as 
well as cardiac sensory signals. Researchers also linked the on-body sensors into a wireless network to monitor the combination of signals as they progress. Additionally, the sensor remains intact on slightly warm water for a couple of days; however, hot water treatment enables easy removal of it. Quite interestingly, eco-friendliness and recyclability provide an extra edge of the device. This proves that there is plenty of scope of directly printing diagnostics schemes on skin which can be reusable as well as washable. As illustrated in Figure 1, these printable sensors can be regarded as the prudent replacements for future. With skin friendly materials, there lies immense potential in implementing these printable sensory schemes for diagnosing various ailments. In the same note, it can also be put on record that this will lessen environmental wastes as well as other allied pollutions; thus boosting the overall ecological balance. As such, it can be easily perceived that there has arisen tremendous interest as well as huge investments towards the development of such wearable biosensors. Since, this field is flourishing; we can apprehend a large surge of selfpowered wireless personalized health monitor. Now, with the recent capability of printable sensors directly on to the skin, it raises the scale bar to new heights with apprehensions of more efficient as well as minimalist sensory approaches which can be conceivable in future course of time.

\section{Funding}

There is no funding for this.

\section{Conflicts of interest}

There is no conflict of interest.

\section{Acknowledgments}

None.

\section{References}

1. Biswas R, Pradhan M. A comparative analysis of all fiber optic sensors for detection of adulteration in fossil fuels. Opt Quant Electron. 2020;52:62.

2. Boruah BS, Gogoi D, Biswas R. Bio-inspired finger like Cu-electrodes as an effective sensing tool for heavy metal ion in aqueous solution. Journal of Electrochemical Society. 2020;167(2).
3. Boruah BS, Biswas R, Ojah N. Bio-inspired localized surface plasmon resonance enhanced Sensing of Mercury through Green synthesized Silver nanoparticle. Journal of Lightwave Technology. 2020;38(7):2086-2091.

4. Biswas R, Saha D. Probing volatile liquid through an electrical sensor with up gradation to a breathalyzer for drunken drivers. Applied Physics A. 2020;126:313.

5. Biswas R, Karmakar PK. An Inexpensive and Novel Optical Scheme of Assessing Adulterants in Emulsions. Biointerface Researh in applied Chemistry. 2020;10(6):6874-6880.

6. Baruah BS, Biswas R, Neog U. Ultrasensitive Trace Determination of Cadmium Through a Green Synthesized Hybrid PVA-Chitosan Nanocomposite. Plasmonics. 2020.

7. Biswas R. Inexpensive Hetero-core Spliced Fiber Optic Setup for Assessing Strain. Sens Imaging. 2020;21:38.

8. Baruah BS, Biswas R, Probing lead ion contamination in aqueous solution through bio-inspired surface modification of gold nanoparticles on D-shaped fiber. IEEE Transactions on Nanotechnology. 2019;18:770 775 .

9. Baruah BS, Biswas R. Mangiferaindica leaf extract mediated gold nanoparticles: a novel platform for sensing of As(III). IEEE Sensors Letter.2019:3(3).

10. Baruah BS, Biswas R, Deb P. A green colorimetric approach towards detection of arsenic (III): a pervasive environmental pollutant. Optics and Laser Technology. 2018;111:825-829.

11. Baruah BS, Biswas R. Localized surface plasmon resonance based U shaped optical fiber probe for the detection of $\mathrm{Pb} 2+$ in aqueous medium. Sensors and Actuators B Chemical. 2018;276:89-94.

12. Paul D, Biswas R. Facile fabrication of sensing set-up for size detection of nanoparticles. IEEE Transactions on Nanotechnology. 2018;17(3):596602.

13. Jiang N, Ali K Yetisen, Nico Linhart, et al. Fluorescent dermal tattoo biosensors for electrolyte analysis. Sensors and Actuators B: Chemical. 2020;320:128378

14. Yetisen AK, et al. Dermal Tattoo Biosensors for Colorimetric Metabolite Detection. Angewandte Chemie. 2019;131(31):10616-10623.

15. Ling Zhang, Hongjun Ji, Houbing Huang, et al. Wearable Circuits Sintered at Room Temperature Directly on the Skin Surface for Health Monitoring. ACS Applied Materials \& Interfaces. 2020;12 (40):45504. 